\title{
Post Keratoplasty Dimensional and Refractive Change in Contact Lens and Spectacle Corrected Cases
}

\author{
E. G. WOODWARD, ${ }^{*}$ L. C. M. MOODALEY,${ }^{* *}$ C. LYONS,${ }^{* *}$ C. DAVISON,${ }^{* *}$ \\ R. J. BUCKLEY, ${ }^{* *}$ V. A. BARBUR ${ }^{* * *}$ \\ London and Oxford
}

\begin{abstract}
Summary
Two groups of patients who had corneal transplantations for keratoconus were analysed retrospectively. One group had been visually corrected with corneal contact lenses and the second group had worn spectacles or used no optical correction.

The reduction in post graft corneal astigmatism with time following removal of sutures was examined by the method of regression in groups.

The results showed that the reduction in corneal astigmatism was significantly greater in the contact lens wearing group.
\end{abstract}

It has long been reported that astigmatism following corneal transplantation decreases with time. It has also been suggested that this reduction may be faster in patients wearing contact lenses as compared with those whose vision is corrected with spectacles.

Reports of corneal curvature following corneal transplantation appear in the literature. ${ }^{1,2,3,4,5}$ However, they relate to measurements of graft curvature at various points in time and do not analyse in a serial manner, changes in individual graft curvature over a period of time following removal of graft sutures.

To test the hypothesis that corneal astigmatism decreases faster in patients wearing contact lenses and to obtain a serial evaluation of changes in graft contour, two groups of patients were analysed retrospectively following corneal transplantation for keratoconus. Group S had spectacles or no correction and Group C were corrected with corneal contact lenses. The method of regression in groups ${ }^{6}$ was used to analyse the meridional differences in corneal curvature with time for the two groups of patients.

\section{Materials and Method}

Patients in the study had all had their transplant operations done at Moorfields Eye Hospital between September 1978 and March 1988 by the surgical team in the Contact Lens and Prosthesis Department.

The criteria for corneal transplantation were intolerance to contact lenses or visual acuity of approximately $6 / 18$ or worse. None of the patients in the study were transplanted for acute hydrops.

From patient records, measurements of meridional corneal difference were extracted at various times following removal of graft sutures.

Keratometry measurements had not always been recorded during the first few months fol-

\footnotetext{
${ }^{*}$ Department of Optometry and Visual Science, City University, London

** Contact Lens \& Prosthesis Department, Moorfields Eye Hospital, London

${ }^{* * *}$ Linacre College, University of Oxford, Oxford

Correspondence to: Department of Optometry and Visual Science, City University, Dame Alice Owen Building, 311-321 Goswell Road, London EC1V 7DD.
} 
Table I Characteristics of Groups $S$ and C

\begin{tabular}{lcc}
\hline & Group $S$ & Group C \\
\hline Mean length of follow-up (mths) & $20+/-12.19$ & $24+/-18.18$ \\
& range $(5-120)$ \\
Mean number of follow-up visits & $3.74+/-0.84$ & $3.70+/-0.17$ \\
Mean corneal astigmatism-1st visit & range (3-6) & range $(3-5)$ \\
& $0.95+/-0.60$ & $0.91+/-0.45$ \\
Mean corneal astigmatism-Last visit & $(0.10-2.30)$ & $(0.29-1.62)$ \\
Reduction in corneal astigmatism (\%) & $0.78+/-0.66$ & $0.68+/-0.32$ \\
\end{tabular}

lowing removal of graft sutures. In these cases, the spectacle refractive astigmatism was converted into corneal curvature by using standard tables. It is appreciated that the astigmatism may not all have been corneal so there is a possible source of error with these measurements. Patients who had undergone refractive surgery for high graft astigmatism or who needed re-suturing were excluded, as were those who had more than one rejection episode.

Graft sutures were removed between 3 and 30 months following corneal transplantation, (Group S; mean $=15.59+/-6.60$; range 3-30: Group C mean $=12.38+/-4.37$; range 6-20).

\section{Results}

The data was first analysed in groups. Group S
(23 eyes) had spectacles or no correction for the period analysed and Group C (20 eyes) were corrected with rigid corneal contact lenses. The mean time of contact lens fitting was approximately three months post removal of graft sutures $(2.95 \mathrm{mths}+/-1.16$; range 1-6 months). (See Table I).

Both groups demonstrated a reduction in corneal astigmatism over the period analysed and the percentage reduction was greater in the contact lens wearing group. (See Table I).

Regression in groups was used to verify this finding. ${ }^{6}$ The model used is based on a parallel least squares regression which yields the best gradient for the combined data within each group (See Figs 1 and 2). The model allows changes in individual graft curvature to be predicted as a function of time (in months). The analysis predicts negative gradients for

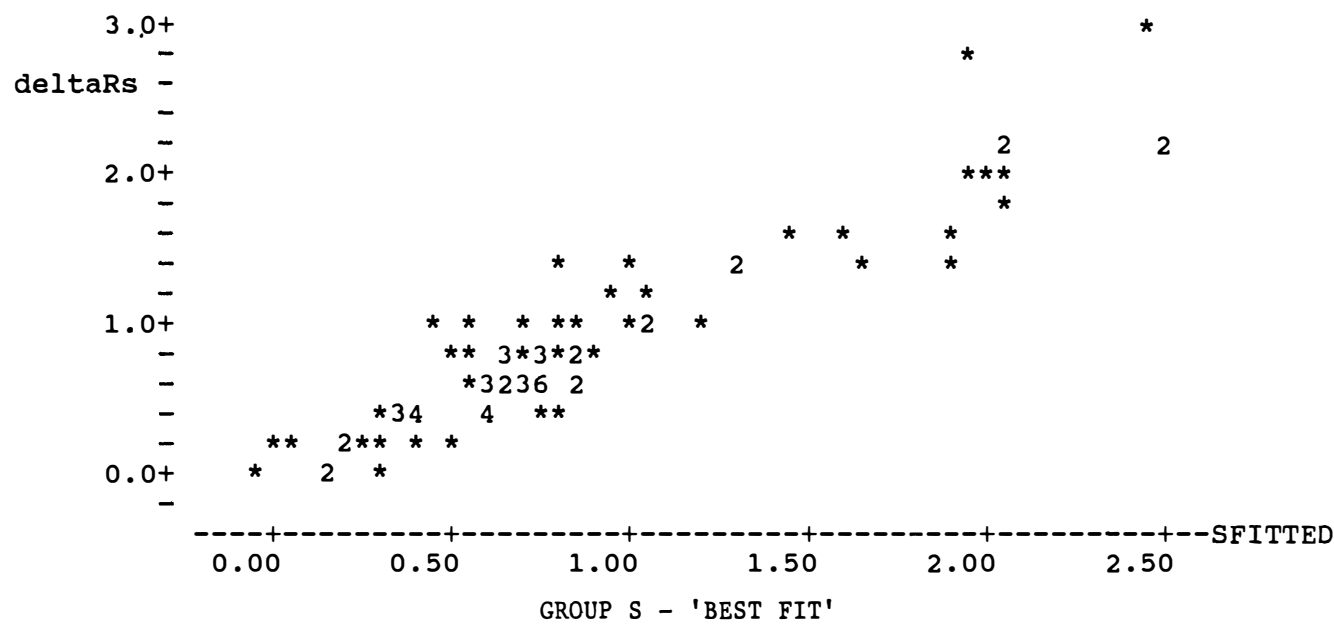

Fig. 1. Data represent measured changes in individual graft curvature for Group $S$ plotted as a function of the changes which are predicted on the basis of the regression model fitted to the grouped data points, i.e., a common negative gradient of -0.0095 which shows that changes in graft curvature decrease with time (in months). 


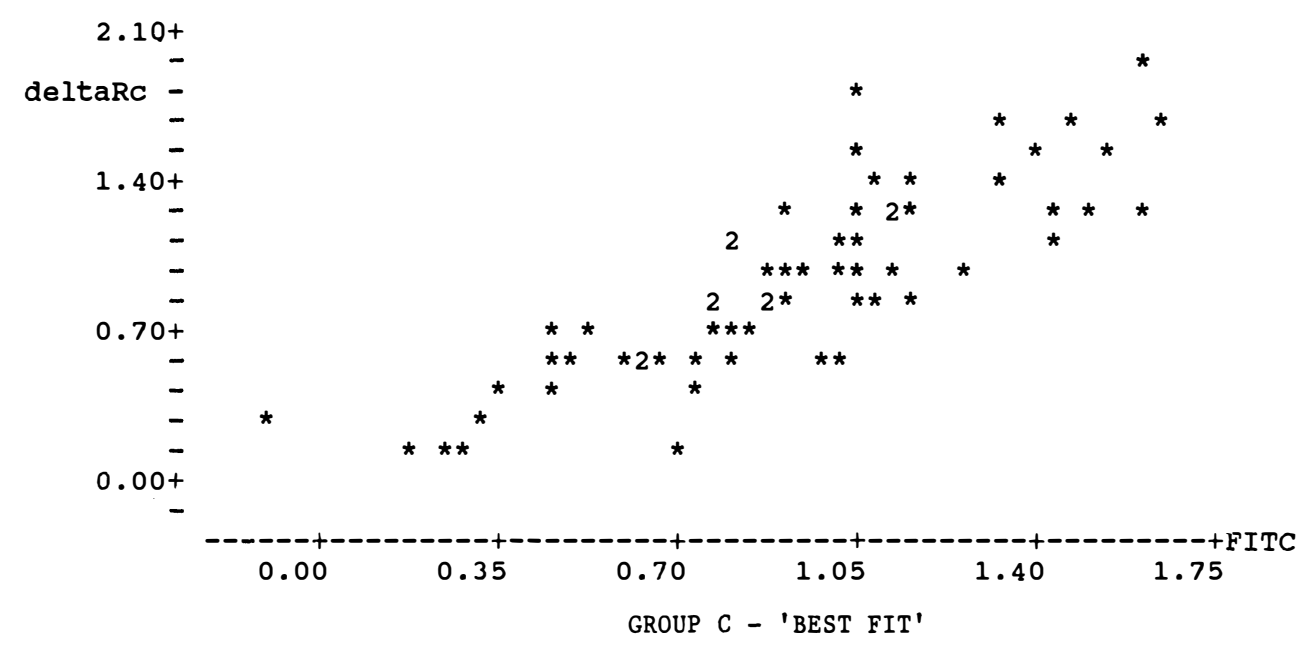

Fig. 2. Similar data to those shown in figure 1 but obtained for sample of patients corrected with rigid corneal contact lenses. The regression model fitted to the grouped data points has a negative gradient of -0.0.0215 which indicates a more rapid decrease in graft curvature changes as a function of time for this category of patient.

each group (Group S -0.0095 and Group C $-0.0215)$, which suggests a decrease in corneal astigmatism with time. The ratio of the two gradients (2.26) suggests that the reduction is greater in the contact lens wearing group.

The regression analysis yields a measure of the variance associated with the best gradient for each group and this provides a means of testing the significance of the difference between the two gradients which describe the change in corneal astigmatism with time for each group. The ' $t$ ' test was applied to determine the significance of the difference. The difference in gradients was found to be significant at the $6 \%$ level.

\section{Discussion}

It was expected that patients with greater degrees of corneal astigmatism were more likely to have contact lens correction, however the mean corneal astigmatism at the first visit was lower in the contact lens wearing group. The choice of contact lens correction was not therefore based upon the amount of astigmatism. Factors influencing the choice were mode of correction of the contralateral eye, a history of successful contact lens wear, the degree of anisometropia and the quality of visual acuity obtainable. These findings support those of Ruben and Colebrook in $1979 .^{3}$
The results show clearly that contact lens correction following corneal transplantation leads to a more rapid reduction in graft astigmatism as compared with uncorrected or spectacle corrected cases.

Even successful clear grafts have been shown to be thicker than the normal cornea, ${ }^{7}$ and this may render them more amenable to the moulding action of the posterior surface of the contact lens. However, it must be noted that measurements were usually taken immediately following contact lens removal and the moulding effect may not be permanent. Were it possible to repeat the measurements some days after contact lens wear, different results may have been obtained. Unfortunately it is very difficult to persuade patients with good contact lens acuity to cease lens wear to obtain these measurements.

The changes in corneal shape demonstrated confirm the clinical finding that post keratoplasty patients frequently need refitting following the removal of graft sutures. In the past it has been suggested that the fitting of contact lenses could be regarded as a technique for the management of post keratoplasty astigmatism. Although a reduction has been shown to occur, it is neither as quick or of the same order of magnitude as that produced by refractive surgical techniques. The greater reduction of corneal astigmatism must 
therefore be regarded as a beneficial consequence of contact lens wear.

\section{References}

${ }^{1}$ Ruben M: Contact lens and keratoplasty curvatures. In Corneal Grafting, 244-80. Edited by T. A. Casey. Butterworth: London. 1972.

${ }^{2}$ Davies PD, Ruben M, Woodward EG: Keratoconus-an analysis of the factors which influence the optical results of keratoplasty. Transactions of the European Contact Lens Society of Ophthalmologists, Ghent 1977, 97-9.

${ }^{3}$ Ruben $M$ and Colebrook E: Keratoconus kerato- plasty curvatures and contact lens wear. $\mathrm{Br} J$ Ophthalmol 1979, 63: 268-73.

${ }^{4}$ Smeddy GE et al: Contact lens or keratoplasty? Ophthalmol 1988, 95: 487-92.

${ }^{5}$ Wilson SE and Bourne WM: Effect of recipientdonor trephine size disparity on refractive error in Keratoconus. Ophthalmol 1989, 96: 299-305.

${ }^{6}$ Armitage P and Berry G: Statistical methods in Medical Research, 2nd Edition publ. 1988. Blackwell Scientific Publications.

${ }^{7}$ Ruben M, Colebrook E, Guillon M: Keratoconus keratoplasty thickness and endothelial morphology. Br J Ophthalmol 1979, 63: 790-3. 